\title{
CONJUGATE LOCI OF TOTALLY GEODESIC SUBMANIFOLDS OF SYMMETRIC SPACES
}

\author{
J. M. BURNS
}

\begin{abstract}
The conjugate and cut loci of fixed point sets of involutions which fix the origin of a compact symmetric space are studied. The first conjugate locus is described in terms of roots and weights of certain representations. When the first conjugate locus and the cut locus agree, we study Morse functions which give a simple decomposition of the symmetric space. We describe for some examples the topological implications of our results.
\end{abstract}

\section{INTRODUCTION}

This paper will be concerned with the study of conjugate and cut loci of fixed point sets of involutions which fix the origin of a compact symmetric space. We will first describe the conjugate loci of these submanifolds and then give a description of their first conjugate loci in terms of roots, and weights of certain representations. We will show that in some cases the first conjugate locus and the cut locus agree. This being the case, we obtain a Morse function which gives a particularly simple decomposition of the symmetric space. We note that by taking our involution to be the symmetry at the origin we can obtain results on the conjugate locus of a point, a subject which has been studied in detail by Wong [W, 1], Sakai [ S, 1], Takeuchi [T, 1], Bott and Samelson [B-S, 1] and Crittenden [Cr, 1] among others. We outline by use of some examples how such a decomposition can be used to calculate homology. The results obtained here comprise part of the author's Ph.D. thesis and he would like to thank Professor T. Nagano for his encouragement and expert guidance. The author would also like to thank the referee for the many helpful suggestions.

\section{PRELIMINARIES}

Definition. A Riemannian manifold $M$ is called a symmetric space, if for each point $q$ of $M$, there exists an involutive isometry $s_{q}$ of $M$ such that $q$ is an isolated fixed point of $s_{q}$. We call $s_{q}$ the symmetry at $q$. If $G$ is the closure in the compact open topology of the group of symmetries generated by $\left\{s_{q} \mid q \in M\right\}$, then it is known that $G$ is transitive on $M$, (provided $M$ is connected) and hence the isotropy subgroup $K$, say at 0 , is compact and $M=G / K$. We will assume throughout that $G$ is semisimple and that $M$ (and therefore $G$ ) is compact. We will denote the Lie algebra of $G$ by $\mathbf{g}$ and the

Received by the editors December 5, 1989 and, in revised form, February 18, 1991.

1980 Mathematics Subject Classification (1985 Revision). Primary 53C35. 
involution $\operatorname{ad}\left(s_{0}\right)(g)=s_{0} g s_{0}$ by $\operatorname{ad}\left(s_{0}\right)$. We will use the same notation for the induced involution on $\mathbf{g}$. Since $G$ is semisimple, the Killing form $B_{\mathbf{g}}$ is a negative definite bilinear form on $\mathbf{g}$ invariant under ad $G$.

The Cartan decomposition of $\mathbf{g}$ with respect to $\operatorname{ad}\left(s_{0}\right)$ is given by $\mathbf{g}=\mathbf{k}+\mathbf{m}$ where $\mathbf{k}$ and $\mathbf{m}$ denote the eigenspaces of plus and minus one respectively. Since $\mathbf{k}$ is the Lie algebra of $K$, we identify $\mathbf{m}$ with the tangent space to $M$ at 0 . The following inclusions are well known.

$$
[\mathbf{m}, \mathbf{m}] \subset \mathbf{k}, \quad[\mathbf{k}, \mathbf{m}] \subset \mathbf{m}, \quad[\mathbf{k}, \mathbf{k}] \subset \mathbf{k} .
$$

We will denote by $\langle$,$\rangle the unique Riemannian metric on M$, which is invariant under $G$ and coincides on the tangent space $T_{0} M$ to $M$ at 0 with $-B_{\mathbf{g}}$. Recall that on a symmetric space $M$ the exponential map Exp: $T_{0} M \rightarrow M$ is given by $\operatorname{Exp} X=(\exp X)(0)$, where exp is the exponential map of $G$, sometimes just written as $e^{X}$, for $X \in \mathbf{m}$. We will denote by $\mathbf{h}$ a maximal abelian subalgebra of $m$ and by $A$ its image under Exp: $T_{0} M \rightarrow M$, that is $A$ is a maximal torus through 0 in $M$. The dimension of such a torus is then by definition the rank of $M$ denoted by $r(M)$. Using the fact that $\left\{\operatorname{ad}(H)^{2} \mid H \in \mathbf{h}\right\}$ is a commutative system of semisimple operators stabilizing $\mathbf{m}$ and $\mathbf{k}$ we get the following well-known result (see [H1, Chapter 7]).

Theorem A. (1) We have the following root space decompositions

$$
\mathbf{m}=\mathbf{h}+\sum_{\alpha \in R(M)} \mathbf{m}_{\alpha}, \quad \mathbf{k}=\mathbf{k}_{0}+\sum_{\alpha \in R(M)} \mathbf{k}_{\alpha} .
$$

(2) For each $\alpha \in R(M)$ we can choose bases $\left\{X_{\alpha}\right\}$ and $\left\{Y_{\alpha}\right\}$ for $\mathbf{m}_{\alpha}$ and $\mathbf{k}_{\alpha}$ respectively such that

$$
\begin{gathered}
{\left[H, X_{\alpha}\right]=\alpha(H) Y_{\alpha} \text { and }\left[H, Y_{\alpha}\right]=-\alpha(H) X_{\alpha} \text { for all } H \in \mathbf{h},} \\
\operatorname{ad}(\exp H) X_{\alpha}=\cos \alpha(H) X_{\alpha}+\sin \alpha(H) Y_{\alpha}
\end{gathered}
$$

and

$$
\operatorname{ad}(\exp H) Y_{\alpha}=\cos \alpha(H) Y_{\alpha}-\sin \alpha(H) X_{\alpha} \quad \text { for all } H \in \mathbf{h} .
$$

Definition. The linear forms $\alpha: \mathbf{h} \rightarrow \mathbb{R}$ are called the roots of $M$ with respect to $\mathbf{h}$ and $\mu(\alpha)=\operatorname{dim} \mathbf{m}_{\alpha}=\operatorname{dim} \mathbf{k}_{\alpha}$ is called the multiplicity of $\alpha$. We can order the roots in a standard manner and this enables us to define positive roots and simple roots. We will denote the simple roots by $\alpha_{1}, \ldots, \alpha_{r}$ where $r=r(M)$ and we will use the symbol $\Sigma$ to denote the set of simple roots.

Definition. A Weyl chamber is a connected component of $\mathbf{h}-\bigcup \operatorname{ker} \alpha$ where the union taken is over all roots of $M$.

The normalizer and centralizer of $\mathbf{h}$ in $K$ will be denoted as follows. $N(\mathbf{h}, K)$ $=\{k \in K \mid \operatorname{ad}(k) \mathbf{h}=\mathbf{h}\}, C(\mathbf{h}, K)=\left\{k \in K \mid \operatorname{ad}(k)_{\mid \mathbf{h}}=1_{\mathbf{h}}\right\}$. The Weyl group will be denoted by $W$ and is defined to be the quotient $N(\mathbf{h}, K) / C(\mathbf{h}, K)$. The following facts can be found in [H1, Chapter 7].

The diagram $\mathscr{D}$ is defined to be the following union of hyperplanes $\mathscr{D}=$ $\bigcup \alpha^{-1}(\pi \mathbb{Z})$ where the union is taken over all $\alpha$. A connected component of $\mathbf{h}-\mathscr{D}$ is called a fundamental cell of $\mathbf{h}$. The closure of the unique fundamental cell containing 0 and contained in the closed positive Weyl chamber will be called the fundamental Weyl simplex and will be denoted by $S$, and $S$ therefore has the following description when $M$ is irreducible. 
$S=\{H \in \mathbf{h} \mid 0 \leq \alpha(H) \leq \pi$ for all $\alpha \in \Sigma \cup \widetilde{\alpha}\}$ where $\widetilde{\alpha}$ denotes the highest root. The following is known [T, 1].

Theorem B. $M=K \operatorname{Exp} S=\operatorname{Exp} \operatorname{ad}(K) S$.

Finally we point out some of the geometric meanings of the roots.

Theorem $C$. If $R$ denotes the Riemannian curvature tensor of $M$, then

$$
R(X, Y) Z(0)=-[[X, Y], Z]
$$

for $X, Y, Z \in \mathbf{m}$ and $\nabla R=0$.

Corollary D. If $H \in h$ and $X \in \mathbf{m}_{\alpha}$ then $R(X, H) H=\alpha(H)^{2} X$.

1

In this section we will give a description of the conjugate locus of certain totally geodesic submanifolds of $M$. We begin by making the necessary definitions and recalling some basic facts. A vector field $V$ along a geodesic $\gamma$ is called a Jacobi field along $\gamma$, if it is the variation vector field of a variation of $\gamma$ through geodesics. This is equivalent to $V$ satisfying the Jacobi equation $\nabla_{\gamma^{\prime}} \nabla_{\gamma^{\prime}} V+R\left(V, \gamma^{\prime}\right) \gamma^{\prime}=0$ where $\nabla_{\gamma^{\prime}}$ denote covariant differentiation in the direction of $\gamma$. We say that $\gamma\left(s_{c}\right)$ is conjugate to $0=\gamma(0)$ if there exists a nontrivial Jacobi field $V$ along $\gamma$ vanishing at 0 and at $\gamma\left(s_{c}\right)$, and we say that the tangent vector $s_{c} \gamma^{\prime}(0)$ at 0 is a tangent conjugate point of 0 . We will assume throughout that geodesics are parametrized by arclength. The set of all tangent conjugate points of 0 is called the tangent conjugate locus of 0 and its image under the exponential map Exp: $T_{0} M \rightarrow M$ is called the conjugate locus of 0 in $M$, denoted by $J L(\{0\}, M)$. If $s_{f}$ is the first parameter value for which $\gamma(s)$ is conjugate to 0 along $\gamma$, then we say that $\gamma\left(s_{f}\right)$ is a first conjugate point of 0 along $\gamma$ and that $s_{f} \gamma^{\prime}(0)$ is a first tangent conjugate point of 0 along $\gamma$. The set of all first tangent conjugate points of 0 is called the first tangent conjugate locus of 0 and it's image under the exponential map Exp: $T_{0} M \rightarrow M$ is called the first conjugate locus of 0 in $M$, denoted by $F L(\{0\}, M)$. Let $\gamma$ be a geodesic normal to an embedded submanifold $N$ of $M$, then following Ambrose [A, 1], we generalize the notion of a conjugate point of 0 along $\gamma$ to that of a conjugate point of $N$ along $\gamma$. In order to do so we consider variation vector fields of variations of $\gamma$ through geodesics initially normal to $N$. This is equivalent to saying that the variation vector field $V$ is initially tangent to $N$ and satisfies the initial condition that $\nabla_{\gamma^{\prime}} V(\gamma(0))-S_{\gamma^{\prime}(0)} V(\gamma(0))$ is normal to $N$, where $S$ is the second fundamental form of $N$. From now on we will usually denote the covariant derivative of $V$ in the direction of $\gamma$ by $V^{\prime}$.

Definition. We say that $x=\gamma\left(s_{c}\right)$ is a conjugate point of $N$ along $\gamma:(\mathbb{R}, 0) \rightarrow$ $(M, N)$ if there exists a nontrivial Jacobi field $V$ along $\gamma$, satisfying the above initial conditions and vanishing at $x$. The conjugate locus of $N$ in $M$ will be denoted by $J L(N, M)$, and is defined as follows,

$J L(N, M)=\{x \in M \mid x$ is conjugate to $N$ along some geodesic $\gamma$ normal to $N\}$.

We say that $x=\gamma\left(s_{f}\right)$ is a first conjugate point of $N$ along $\gamma$ if there exists a nontrivial Jacobi field satisfying the above initial conditions vanishing at $x$, 
but no such Jacobi field along $\gamma$ vanishes at $\gamma(s)$ for $s \in\left(0, s_{f}\right)$. The first conjugate locus of $N$ in $M$ will be denoted by $F L(N, M)$ and is defined as follows,

$F L(N, M)=\{x \in M \mid x$ is a first conjugate point of $N$ along some geodesic $\}$.

Let $t$ be an involutive isometry of $M$ such that $t(0)=0$, and let $M^{t}$ be the component of the fixed point set of $t$ through 0 , that is $M^{t}=F(t, M)(0)$, then by a well-known theorem of Kobayashi $[\mathrm{K}, 1], M^{t}$ is a totally geodesic submanifold of $M$ and is therefore a symmetric space. Let $G^{t}$ be the identity component of the fixed point set of $\operatorname{ad}(t)$ in $G$, that is $G^{t}=F(\operatorname{ad}(t), G)_{(1)}$, then $F(\operatorname{ad}(t), G)$ contains all the symmetries $s_{x}$ for $x \in M^{t}$, since if $x$ in $M^{t}$, we have that $s_{x}(x)=x$ and $t(x)=x$, also on $T_{x} M d s_{x}=-1$ therefore $d s_{x} \circ d t=d t \circ d s_{x}$ which implies that $d\left(s_{x} \circ t\right)=d\left(t \circ s_{x}\right)$. We now have that $s_{x} \circ t$ and $t \circ s_{x}$ are isometries fixing $x$ which agree on $T_{x} M$ so $s_{x} \circ t=t \circ s_{x}$ on $M$ (since $M$ is connected). We have therefore since $G^{t}$ is closed, that $G^{t}$ is transitive on $M^{t}$ and hence $M^{t}=G^{t} / K^{t}$ where $K^{t}$ is the typical isotropy subgroup at 0 say. If $\mathbf{g}=\mathbf{k}+\mathbf{m}$ is the Cartan decomposition with respect to $\operatorname{ad}\left(s_{0}\right)$, then since $\operatorname{ad}\left(s_{0}\right)$ and $\operatorname{ad}(t)$ commute by above and we see that $\operatorname{ad}\left(s_{0}\right) \circ \operatorname{ad}(t)(X)=\operatorname{ad}(t) \circ \operatorname{ad}\left(s_{0}\right)(X)= \pm \operatorname{ad}(t)(X)$. Thus ad $(t)$ stabilizes the plus and minus one eigenspaces $\mathbf{k}$ and $\mathbf{m}$ of $\operatorname{ad}\left(s_{0}\right)$. We can therefore decompose $\mathbf{m}$ and $\mathbf{k}$ into their eigenspaces with respect to the involution $\operatorname{ad}(t)$, so that we have $\mathbf{m}=\mathbf{m}^{t}+\mathbf{m}^{-t}$ and $\mathbf{k}=\mathbf{k}^{t}+\mathbf{k}^{-t}$ (the sign convention being the obvious one). Letting $\mathbf{g}^{t}$ denote the Lie algebra of $G^{t}$, we have that $\mathbf{g}^{t}=\mathbf{k}^{t}+\mathbf{m}^{t}$ and we can identify $\mathrm{m}^{t}$ with the tangent space $T_{0} M^{t}$ to $M^{t}$ at 0 . We can also identify $\mathbf{m}^{-t}$ with the tangent space $T_{0} M^{-t}$ to $M^{-t}$ at 0 , where $M^{-t}=$ $F\left(s_{0} \circ t, M\right)(0)$, also a symmetric space.

Lemma 1.1. We have the following orthogonal decompositions.

$$
\mathbf{m}^{t}=\sum_{\alpha \in R_{t}} \mathbf{m}_{\alpha}^{t}, \quad m^{-t}=\sum_{\alpha \in R_{-t}} \mathbf{m}_{\alpha}^{-t}
$$

where $R_{t}$ and $R_{-t}$ are sets of linear forms on $\mathbf{h}^{-t}$ a maximal abelian subalgebra of $\mathbf{m}^{-t}$, which are not necessarily distinct and may include the zero form.

Proof. Let $H \in \mathbf{h}^{-t} \subset \mathbf{m}^{-t}$, then $\operatorname{ad}(H) \mathbf{m}^{-t}=\left[H, \mathbf{m}^{-t}\right] \subset[\mathbf{m}, \mathbf{m}] \subset \mathbf{k}$ and $\operatorname{ad}(t)\left[H, \mathbf{m}^{-t}\right]=\left[\operatorname{ad}(t) H, \operatorname{ad}(t) \mathbf{m}^{-t}\right]=\left[-H,-\mathbf{m}^{-t}\right]=\left[H, \mathbf{m}^{-t}\right]$. Therefore by definition of $\mathbf{k}^{t}$ we have that $\operatorname{ad}(H) \mathbf{m}^{-t} \subset \mathbf{k}^{t}$. A similar argument shows that $\operatorname{ad}(H) \mathbf{k}^{t} \subset \mathbf{m}^{-t}$ and therefore we have that the commutative system of semisimple operators $\left\{\operatorname{ad}(H)^{2} \mid H \in \mathbf{h}^{-t}\right\}$ stabilizes $\mathbf{m}^{t}$ and $\mathbf{m}^{-t}$. Note we also get the following decompositions of $\mathbf{k}^{t}$ and $\mathbf{k}^{-t}$.

$$
\mathbf{k}^{t}=\sum_{\alpha \in R_{-t}} \mathbf{k}_{\alpha}^{t}, \quad \mathbf{k}^{-t}=\sum_{\alpha \in R_{t}} \mathbf{k}_{\alpha}^{-t} .
$$

This follows from the fact that $\operatorname{ad}(H): \mathbf{m}_{\alpha}^{t} \rightarrow \mathbf{k}_{\alpha}^{-t}$ is a linear isomorphism if $\alpha \in R_{-t}$ and $\alpha(H) \neq 0$ and similarly for $\operatorname{ad}(H): \mathbf{m}_{\alpha}^{-t} \rightarrow \mathbf{k}_{\alpha}^{t}$ with $\alpha \in R_{t}$.

Before proceeding we recall a few more facts. For $X \in \mathbf{g}$ we may consider $X$ as a vector field on $M$, which we will also denote by $X$. It's value $X(p)$ at $p \in M$ is the initial tangent to the curve $(\exp t X)(p)$. Since $G$ acts on $M$ as a group of isometries, it therefore carries geodesics to geodesics and hence $X$ restricted to a geodesic is a Jacobi field along that geodesic. 
Given a compact Lie transformation group $L$ acting on a manifold $M$, on which we have an $L$ invariant metric, the orbit of $L$ through $x$ will be denoted by $L(x)$. The orbit $L(x)$ is a compact submanifold homeomorphic with $L / L_{x}$ where $L_{x}$ is the isotropy subgroup at $x$.

Definition. An orbit whose dimension is less than that of a maximal dimensional orbit will be called a singular orbit.

Theorem 1.2. The conjugate locus of $M^{t}$ in $M$ is the union of the singular $G^{t}$ orbits, that is $J L\left(M^{t}, M\right)=\left\{x \in M \mid G^{t}(x)\right.$ is singular $\}$.

Proof. Suppose $x \in J L\left(M^{t}, M\right)$, we may assume that $x$ lies in a maximal torus in $M^{-t}$ and that $x$ is a conjugate point of $M^{t}$ along a geodesic $\gamma=$ $\gamma_{H}: s \rightarrow \operatorname{Exp} s H$ issuing from 0 with $H \in \mathbf{h}^{-t}$. We wish to consider Jacobi fields $V$ along $\gamma$ for which $V(\gamma(0)) \in T_{0} M^{t}$ and $V^{\prime}(\gamma(0))-S_{\gamma^{\prime}} V(\gamma(0))$ is normal to $M^{t}$ and therefore is contained in $T_{0} M^{-t}$. Since $M^{t}$ is totally geodesic, the second fundamental form of $M^{t}$ is zero and hence the second initial condition reduces to the condition $V^{\prime}(\gamma(0)) \in T_{0} M^{-t}$. The set of such Jacobi fields which vanish for some parameter value $s_{c}>0$ will be denoted by $\mathscr{J}^{\gamma}$, that is $\mathcal{J}^{\gamma}$ is the set of all Jacobi fields satisfying the following conditions.

$$
V(\gamma(0)) \in T_{0} M^{t}, \quad V^{\prime}(\gamma(0)) \in T_{0} M^{-t}, \quad \text { and } \quad V\left(s_{c}\right)=0 .
$$

We will show that all Jacobi fields in $\mathcal{J}^{\gamma}$ are the restrictions to $\gamma$ of vector fields in $\mathbf{g}^{t}$. We define $V_{0}: \mathbb{R} \rightarrow T_{0} M$ by $V_{0}(s)=$ the parallel transport to 0 along $\gamma$ of $V(\gamma(s))$. Since the curvature tensor is parallel we may identify $V$ with $V_{0}$ and consider the Jacobi equation as an equation in $T_{0} M$. Since $R\left(X_{\alpha}, H\right) H=$ $\alpha(H)^{2} X_{\alpha}$ at 0 , we see that an element $V$ of $\mathscr{J}^{\gamma}$ can be considered as a solution of the system of equations,

$$
\left(V_{\alpha}^{ \pm}\right)^{\prime \prime}+\alpha(H)^{2} V_{\alpha}^{ \pm}=0 \quad \text { where } V=\sum_{\alpha \in R_{t}} V_{\alpha}^{+}+\sum_{\alpha \in R_{-t}} V_{\alpha}^{-}
$$

is the decomposition of $V$ with respect to the decompositions of $\mathbf{m}^{t}$ and $\mathbf{m}^{-t}$. In addition the initial conditions $\left(V_{\alpha}^{+}\right)^{\prime}(0)=0$ and $V_{\alpha}^{-}(0)=0$ must be satisfied. We consider three cases.

Case 1. $\alpha(H)=0$. Then $V_{\alpha}^{ \pm}(s)=V_{\alpha}^{ \pm}(0)+s\left(V_{\alpha}^{ \pm}\right)^{\prime}(0)$, but since $\left(V_{\alpha}^{+}\right)^{\prime}(0)=0$ we have that $V_{\alpha}^{+}(s)=V_{\alpha}^{+}(0)$. However since $V_{\alpha}^{+}\left(s_{c}\right)=0$ for some $s_{c}>0$ we have that $V_{\alpha}^{+}$must be identically zero. $V_{\alpha}^{-}(0)=0$ implies that $V_{\alpha}^{-}(s)=$ $s\left(V_{\alpha}^{-}\right)^{\prime}(0)$, therefore $V_{\alpha}^{-}\left(s_{c}\right)=0$ implies that $\left(V_{\alpha}^{-}\right)^{\prime}(0)=0$ and we have that $V_{\alpha}^{-}$is identically zero being a solution of a second order linear differential equation with both initial conditions equal to zero.

Case 2. $\alpha(H) \neq 0$ and $\alpha \in R_{t}$. In this case we choose $X_{\alpha} \in m_{\alpha}^{t}$ such that $V_{\alpha}^{+}(0)=X_{\alpha}$. Now $\left(X_{\alpha}\right)_{\mid \gamma}^{\prime}(0)=\left[H, X_{\alpha}\right]+\nabla_{X_{\alpha}} H(0)=\left[H, X_{\alpha}\right]$ since $\nabla X=0$ at 0 for $X \in \mathbf{m}$. Since $\left[H, X_{\alpha}\right] \in \mathbf{k}$ we have that $\left(X_{\alpha}\right)_{\mid \gamma}^{\prime}(0)=0$, as the vector fields from $\mathbf{k}$ all vanish at 0 . We therefore have that $X_{\alpha \mid \gamma}=V_{\alpha}^{+}$since they satisfy the same initial conditions.

Case 3. $\alpha(H) \neq 0$ and $\alpha \in R_{-t}$. Since $\left(V_{\alpha}\right)^{\prime}(0) \in \mathbf{m}_{\alpha}^{-t}$ and $\alpha(H) \neq 0$ there exists a unique vector $Y_{\alpha} \in \mathbf{k}_{\alpha}^{t}$ such that $\left(Y_{\alpha}\right)^{\prime}(0)=\left[H, Y_{\alpha}\right]=\left(V_{\alpha}^{-}\right)^{\prime}(0)$ (the first equality holds because $\left.Y_{\alpha}(0)=0\right)$. Also $V_{\alpha}^{-}(0)=0$ implies that $Y_{\alpha \mid \gamma}=$ $V_{\alpha}^{-}$since they have the same initial conditions. We have shown therefore that 
every element of $\mathscr{J}^{\gamma}$ comes from $\mathbf{g}^{t}$, and thus if $x$ is a conjugate point of $M^{t}$ the dimension of the Lie algebra of the isotropy subgroup at $x$ is greater than $\operatorname{dim}\left(k_{0}^{t}+m_{0}^{t}\right)$. From the formula $\operatorname{dim} G^{t}(x)=\operatorname{dim} G^{t}-\operatorname{dim} L\left(G_{x}^{t}\right)$ where $L\left(G_{x}^{t}\right)$ denotes the Lie algebra of the isotropy subgroup at $x$, and the fact that an orbit of maximal dimension has dimension given by $\operatorname{dim} G^{t}(x)=\operatorname{dim} G^{t}-$ $\operatorname{dim}\left(\mathbf{k}_{0}^{t}+\mathbf{m}_{0}^{t}\right)$, we see that $G^{t}(x)$ is singular.

Conversely if $G^{t}(x)$ is singular then $\operatorname{dim} G^{t}(x)<\operatorname{dim} G^{t}-\operatorname{dim}\left(\mathbf{k}_{0}^{t}+\mathbf{m}_{0}^{t}\right)$ and therefore for some $\alpha \neq 0, \alpha \in R_{t} \cup R_{-t}$ there is a $Y_{\alpha} \in \mathbf{m}_{\alpha}^{t}+\mathbf{k}_{\alpha}^{t}$ such that $Y_{\alpha}(x)=0$ and $Y_{\alpha} \neq 0$. Since there is a dense set of directions in $\mathbf{h}^{-t}$ from 0 to $x$, there exists some $H \in \mathbf{h}^{-t}$ such that $\alpha(H) \neq 0, \operatorname{Exp} H=x, Y_{\alpha \mid \gamma}$ is not identically zero along $\gamma=\operatorname{Exp} t H$, and $Y_{\alpha}(x)=0$. This shows that $x$ is conjugate to $M^{t}$ along a geodesic $\gamma$ and thus is contained in the conjugate locus of $M^{t}$ in $M$.

We note that for the special case where $t=s_{0}$ the above theorem gives the variational completeness of Bott and Samelson [B-S, 1].

Corollary 1.3. We have the following description of the set $\mathcal{J}^{\gamma}, \gamma=\operatorname{Exp} t H$,

$\mathscr{J}^{\gamma}=\left\{\left(\sum_{\substack{\alpha \in R_{t} \\ \alpha(H) s_{c} \in \pi / 2(\mathbb{Z}-2 \mathbb{Z})}} X_{\alpha}+\sum_{\substack{\alpha \in R_{-t} \\ \alpha(H) s_{c} \in \pi(\mathbb{Z}-0)}} Y_{\alpha}\right)_{\mid \gamma}: X_{\alpha} \in \mathbf{m}_{\alpha}^{t}, Y_{\alpha} \in \mathbf{k}_{\alpha}^{t}, s_{c}>0\right\}$.

Proof. This follows immediately from the explicit descriptions of the Jacobi fields $X_{\mid \gamma}$ and which are given as follows.

$$
\begin{gathered}
X_{\mid \gamma}=\cos (\alpha(H) s) X, \quad X \in \mathbf{m}_{\alpha}^{t}, \alpha \in R_{t}, \alpha(H) \neq 0, \\
Y_{\mid \gamma}=\sin (\alpha(H) s)[H, Y], \quad Y \in \mathbf{k}_{\alpha}^{t}, \alpha \in R_{-t}, \alpha(H) \neq 0 .
\end{gathered}
$$

Corollary 1.4. The conjugate locus of 0 in $M$ is the union of the singular $K$ orbits, that is $J L(\{0\}, M)=\{x \in M \mid K(x)$ is singular $\}$.

Proof. Take the involution $t$ to be $s_{0}$, then $M^{t}=F\left(s_{0}, M\right)(0)=\{0\}, G^{t}=$ $F\left(\operatorname{ad}\left(s_{0}\right), G\right)_{(1)}=K_{(1)}$ and now apply Theorem 1.2.

\section{Proposition 1.5.}

$$
\operatorname{dim} K(x)=\sum_{\alpha(H) \notin \pi \mathbb{Z}} \operatorname{dim} \mathbf{m}_{\alpha}=\sum_{\alpha(H) \notin \pi \mathbb{Z}} \operatorname{dim} \mathbf{k}_{\alpha}
$$

where $H \in \mathbf{h}, e^{H}(0)=x$, and $\alpha \in R(M)$.

Proof. $Y\left(e^{H}(0)\right)=0$ if and only if $e^{H}\left(e^{-H} Y e^{H}\right)(0)=0$. Since $e^{H}$ is a diffeomorphism, this is equivalent to the saying that $e^{-H} Y e^{H}(0)=0$, that is $\operatorname{ad}\left(e^{-H}\right) Y \in \mathbf{k}$. Now writing $Y=Y_{0}+\sum Y_{\alpha}$ where $Y_{0} \in \mathbf{k}_{0}, Y_{\alpha} \in \mathbf{k}_{\alpha}$, and $\alpha \in R(M)$, we see by Theorem A that $Y$ vanishes at $x$ if and only if $\operatorname{ad}\left(e^{-H}\right) Y=\sum \cos \alpha(H) Y_{\alpha}-\sin \alpha(H) X_{\alpha}$ is a member of $\mathbf{k}$, that is $\alpha(H) \in \pi \mathbb{Z}$ for $X_{\alpha}, Y_{\alpha} \neq 0$.

Recalling the definition of the fundamental Weyl simplex $S$ for an irreducible $M$, we see that its boundary $\operatorname{bd}(S)$ is contained in the union of the hyperplanes $\alpha^{-1}(0), \alpha \in \Sigma$, and $(\widetilde{\alpha})^{-1}(\pi)$. Writing $S$ as a disjoint union $S=\bigcup_{i \in I} S^{i}$ of it's open cells $S^{i}$, we see that vectors in the top dimensional 
cell do not sit on any of the above hyperplanes, vectors in a given $r-1 \mathrm{di}$ mensional cell sit on one hyperplane, vectors in a given $r-2$ dimensional cell sit on the same two hyperplanes and so forth. Since $M=K \operatorname{Exp} S$ and since the dimension of an orbit $K(x)$ depends only on the hyperplanes on which $H$ sits, where $\operatorname{Exp} H=x$ (by Proposition 1.5), we see that the dimensions of the orbits $K(x)$ are the same for all $x \in M^{i}=K \operatorname{Exp} S^{i}$ and are therefore all locally diffeomorphic. Recall the following known result see [BR, 1].

Proposition 1.6. Let $K$ be a compact Lie transformation group acting on a manifold $M$, then for all $x \in M$ there exists a $K$ invariant neighbourhood $U$ of $K(x)$ such that $U$ is a disc bundle over $K(x)$, and $U$ is associated to the principal bundle $K_{x} \rightarrow K \rightarrow K(x)$.

Note from Corollary 1.4 and Proposition 1.5 we have that $J L(\{0\}, M)=$ $K \operatorname{Expbd}(S)$ where $\operatorname{bd}(S)$ is the boundary of the simplex $S$.

Using Proposition 1.6 and the discussion prior to it, we get the following sketch proof of a result due to Takeuchi [T, 1] and also Sakai [S, 1].

\section{Proposition 1.7.}

$$
M=\bigcup_{i \in I} M^{i} \text { and } J L(\{0\}, M)=\bigcup_{i \in I-i_{0}} M^{i}
$$

where each $M^{i}$ is a connected submanifold and $i_{0}$ is the index corresponding to the top dimensional cell of $S . \operatorname{dim} M^{i} \leq \operatorname{dim}^{i} M-2$ for all $i \in I-i_{0}$ and each $M^{i}$ is diffeomorphic with a vector bundle over a compact manifold.

We now apply Theorem 1.2 to obtain descriptions of the conjugate loci of two very important classes of totally geodesic submanifolds. We will first give some basic facts about these submanifolds, for an indepth study and classification see [N-C, 1]. For each smoothly closed geodesic $c$ through 0 , we consider the antipodal point $p$ of 0 on $c$. Denote by $M^{+}(p)$ the orbit $K_{(1)}(p)$, then $M^{+}(p)=F\left(s_{0}, M\right)(p)$ is a symmetric space. Note that $s_{0}$ fixes the point $p$ and therefore $s_{0} \circ s_{p}=s_{p} \circ s_{0}$, so that $\operatorname{ad}\left(s_{p}\right)$ stabilizes $\mathbf{k}$ and $\mathbf{m}$ giving us a Cartan decomposition $\mathbf{g}=\left(\mathbf{k}^{+}+\mathbf{m}^{+}\right)+\left(\mathbf{k}^{-}+\mathbf{m}^{-}\right)$at $p . M^{+}(p)=$ $K / K^{+}$and the tangent space to $M^{+}(p)$ at $p$ can be identified with $\mathbf{k}^{-}$, that is $T_{p} M^{+}(p)=\left\{Y(p) \mid Y \in \mathbf{k}^{-}\right\}$. The normal space to $T_{p} M^{+}(p)$ is the tangent space to another connected totally geodesic submanifold denoted by $M^{-}(p)$. $M^{-}(p)=F\left(s_{p} \circ s_{0}, M\right)(p)=F\left(s_{p} \circ s_{0}, M\right)(0) . T_{0} M^{-}(p)$ can be identified with $\mathbf{m}^{-}$and similarly at $p . M^{-}(p)=G^{-} / K_{p}$ where $G^{-}$is the connected subgroup of $G$ given by the Lie subalgebra $\mathbf{k}^{+}+\mathbf{m}^{-}$and $K_{p}$ is the isotropy subgroup of $p$ in $G^{-}$. One very useful property of $M^{-}(p)$ is that it has the same rank as $M$. Applying Theorem 1.2 using $p$ as origin instead of $0, t=s_{0}$, $G^{t}=F\left(\operatorname{ad}\left(s_{0}\right), G\right)_{(1)}=K_{(1)}$ and $t=s_{p} \circ s_{0}, G t=F\left(\operatorname{ad}\left(s_{0} \circ s_{p}\right), G\right)_{(1)}=G^{-}$we get the following propositions.

Proposition 1.8. $J L\left(M^{+}(p), M\right)=\{x \in M \mid K(x)$ is singular $\}$.

Corollary 1.9. $J L\left(M^{+}(p), M\right)=J L(\{0\}, M)$ and therefore we obtain the stratification of Proposition 1.7 for the conjugate locus of $M^{+}(p)$ in $M$.

Proposition 1.10. $J L\left(M^{-}(p), M\right)=\left\{x \in M \mid G^{-}(x)\right.$ is singular $\}$. 
In this section we will consider the first conjugate locus of a polar set $M^{+}(p)$, where $M$ is assumed to be irreducible.

Lemma 2.1. Let $H_{i} \in \mathbf{h}$ be defined by $\alpha_{k}\left(H_{i}\right)=\pi \delta_{i k}$ for all $\alpha_{k} \in \Sigma$, and let $\tilde{\alpha}=\Sigma n_{i} \alpha_{i}$ be the expression of the highest root of $M$ in terms of the simple roots, then every polar set $M^{+}(p)$ can be described in one of the following ways:

1. $M^{+}(p)=K \operatorname{Exp} 1 / 2 H_{i}$ with $n_{i}=1$ or 2 and $p=\operatorname{Exp} 1 / 2 H_{i}$.

2. $M^{+}(p)=K \operatorname{Exp} 1 / 2\left(H_{t}+H_{s}\right)$ with $n_{t}=n_{s}=1$ and $p=\operatorname{Exp} 1 / 2\left(H_{t}+H_{s}\right)$.

Proof. Since $M=K \operatorname{Exp} S$, a given $M^{+}(p)$ can be described as the $K$ orbit of the image of a vector $H$ in $S$ under the exponential map Exp: $T_{0} M \rightarrow$ $M$. By definition of the polar sets, this point $p$ must be antipodal to 0 on a circle (that is a smoothly closed geodesic) $c$. We therefore have that $\operatorname{Exp}(2 H) \in K$. This implies that $\operatorname{ad}(\exp 2 H) \mathbf{k}=\mathbf{k}$ and $\operatorname{ad}(\exp 2 H) \mathbf{m}=\mathbf{m}$, which since $\operatorname{ad}(\exp 2 H) X_{\alpha}=\cos \alpha(2 H) X_{\alpha}-\sin \alpha(2 H) Y_{\alpha}$ for $X_{\alpha} \in m_{\alpha}$ implies that $\alpha(2 H) \in \pi \mathbb{Z}$ for all $\alpha \in R(M)$. Since $H \in S$ we need only consider two cases.

Case I. $0<\tilde{\alpha}(H)<\pi$, this means that $0<\tilde{\alpha}(2 H)<2 \pi$ and therefore $\widetilde{\alpha}(2 H)=\pi$ and $\widetilde{\alpha}(H)=\pi / 2$. From this it follows that the only possible choices for $H$ are those vectors $1 / 2 H_{i}$ such that $\alpha_{k}\left(H_{i}\right)=\pi \delta_{i k}$ and $n_{i}=1$.

Case II. $\widetilde{\alpha}(H)=\pi$, in this case the only possibilities for $H$ are $H=1 / 2 H_{i}$ with $n_{i}=2$ or $H=1 / 2\left(H_{t}+H_{s}\right)$ with $n_{t}=n_{s}=1$.

Recall now the definition of the bottom space of $M$ denoted by $M^{*}$. The adjoint group of $G$ is defined by $\operatorname{ad} G=G / C$ where $C$ is the centralizer of $G_{(1)}, M^{*}$ is then defined to be $\operatorname{ad} G / F\left(\operatorname{ad}\left(s_{0}\right), \operatorname{ad} G\right)=\operatorname{ad} G / K^{*} . M^{*}$ is characterized by the property that every space locally isometric to $M$ is a covering Riemannian manifold of $M^{*}$. In particular there is a locally isometric projection $\pi: M \rightarrow M^{*}$. We have also that $K^{*}$ is its own normalizer in ad $G$, and therefore the following three conditions are equivalent on $M^{*}$.

(1) $\operatorname{ad}(b) \mathbf{m}=\mathbf{m},(2) \operatorname{ad}(b) \mathbf{k}=\mathbf{k}$, (3) $b \in K^{*}$.

In particular if $\operatorname{ad}(\exp 2 H) \mathbf{k}=\mathbf{k}$ on $M^{*}$ then $(\exp H)(0)$ is antipodal to 0 . Therefore, we have that the converse of the last result is true on $M^{*}$, and we have the following proposition.

Proposition 2.2. If $M=M^{*}$, then $K \operatorname{Exp} 1 / 2 H_{i}$ is a polar set if $n_{i}=1$ or 2 , or else it is the origin 0 . If $n_{t}=n_{s}=1$ then $K \operatorname{Exp~} 1 / 2\left(H_{t}+H_{s}\right)$ is a polar set or else it is the origin 0 .

Recall when we take $t=s_{0}$ and origin at $p, M^{t}=M^{+}(p)$ and $M^{-t}=$ $M^{-}(p)$ and we denote $R_{ \pm t}$ by $R_{ \pm}$. The fact that $M^{-}(p)$ has the same rank as $M$ means we may assume that the maximal torus of $M$ is contained in $M^{-}(p)$, and therefore the decompositions of Lemma 1.1 imply that

$$
\mathbf{m}^{-}=\mathbf{h}+\sum_{\alpha \in R_{-}} \mathbf{m}_{\alpha}^{-}, \quad \mathbf{m}^{+}=\sum_{\alpha \in R_{+}} m_{\alpha}^{+}
$$

where $R_{+}$and $R_{-}$are subsets of the roots of $M$ and $R(M)=R_{+} \cup R_{-}$. As yet it is not clear that $R_{+} \cap R_{-}=\varnothing$, as part of a root space may lie in $\mathbf{m}^{+}$and the rest of it in $\mathbf{m}^{-}$. We now show that this cannot occur. 
Proposition 2.3. $\alpha \in R_{+}$if and only if $\alpha\left(H_{0}\right) \in \pi / 2(\mathbb{Z}-2 \mathbb{Z})$ and $\alpha \in R_{-}$if and only if $\alpha\left(H_{0}\right) \in \pi \mathbb{Z}$, where $M^{+}(p)=k \operatorname{Exp} H_{0}$ and $H_{0} \in S$.

Proof. $\mathbf{k}^{+}=\mathbf{k}_{0}+\sum_{\alpha \in R_{-}} \mathbf{k}_{\alpha}^{+}$and $M^{+}(p)=K / K^{+}$so $Y_{\alpha} \in \mathbf{k}^{+}$if and only if $Y_{\alpha}(p)=0$ where $p=\operatorname{Exp} H_{0}$, that is $Y_{\alpha}\left(\left(\exp H_{0}\right)(0)\right)=0$. This is equivalent to the condition that $\exp H_{0}\left(\exp -H_{0} Y_{\alpha} \exp H_{0}\right)(0)=0$, which since $\exp H_{0}$ is a diffeomorphism is equivalent to the condition that $\left(\exp -H_{0} Y_{\alpha} \exp H_{0}\right)(0)=0$, that is $\operatorname{ad}\left(\exp -H_{0}\right) Y_{\alpha} \in \mathbf{k}$. By Theorem $\mathrm{A}$ we have the following equation, $\operatorname{ad}\left(\exp -H_{0}\right) Y_{\alpha}=\cos \alpha\left(H_{0}\right) Y_{\alpha}-\sin \alpha\left(H_{0}\right) X_{\alpha}$ and therefore the condition that $\alpha \in R_{-}$reduces to the condition that $\alpha\left(H_{0}\right) \in \pi \mathbb{Z}$. Since exp $-H_{0}$ carries $M^{+}(p)$ to $F\left(s_{p}, M\right)(0)$ and since the tangent space to $M^{+}(p)$ is given by $\left\{Y_{\alpha} \mid Y_{\alpha} \in \mathbf{k}_{\alpha}^{-}, \alpha \in R_{+}\right\}$, and the tangent space to $F\left(s_{p}, M\right)(0)$ is given by $\mathbf{m}^{+}$, we see that $\alpha \in R_{+}$if and only if $\operatorname{ad}\left(\exp -H_{0}\right) Y_{\alpha} \in \mathbf{m}_{\alpha}^{+}$. Again by Theorem A we have that $\operatorname{ad}\left(\exp -H_{0}\right) Y_{\alpha}=\cos \alpha\left(H_{0}\right) Y_{\alpha}-\sin \alpha\left(H_{0}\right) X_{\alpha}$ and therefore the condition that $\alpha \in R_{+}$is equivalent to the condition that $\alpha\left(H_{0}\right) \in \pi / 2(\mathbb{Z}-2 \mathbb{Z})$. From these two conditions it is now apparent that $R_{+} \cap R_{-}=\varnothing$.

We now give a description of the first conjugate locus of an $M^{+}(p)$ on the bottom space in the case where the linear isotropy representation of $G^{-}$is absolutely irreducible on the tangent space of $G / G^{-}$. A description can be given in the other cases using the same type of analysis but it needs a case by case argument. We will also assume for simplicity that the root system of $M$ is not $A_{r}$ or that of $E_{6}$ and therefore the $M^{+}(p)$ comes from a $1 / 2 H_{i}$ with $n_{i}=1$ or 2 .

Theorem 2.4. Under the above assumptions the first conjugate locus of a polar set $M^{+}(p)$ is given as follows. 1 .

(1) $F L\left(M^{+}(p), M\right)=K \operatorname{Exp}_{p}\left\{(\widetilde{\alpha})^{-1}(\pi / 2) \cap C_{p}\right\}$ if $p=\operatorname{Exp} 1 / 2 H_{i}$ and $n_{i}=$

(2) $F L\left(M^{+}(p), M\right)=K \operatorname{Exp}_{p}\left\{(\widetilde{\beta})^{-1}(\pi / 2) \cap C_{p}\right\}$ if $p=\operatorname{Exp} 1 / 2 H_{i}$ with $n_{i}=2$, where $\widetilde{\alpha}$ is the highest root of $M, \widetilde{\beta}$ is the highest root in $R_{+}, C_{p}$ is a closed positive Weyl chamber at $p$ and $\operatorname{Exp}_{p}: T_{p} M \rightarrow M$ is the exponential map at $p$.

Proof. We apply Theorem 1.2 taking $p$ as our origin and $t=s_{0}$. We then have $M^{t}=M^{+}(p), G^{t}=K_{(1)}$, and $g^{t}=k^{+}+k^{-}$. Corollary 1.3 tells us that

$\mathscr{J}_{H}^{\gamma}=\left\{\left(\sum_{\substack{\alpha \in R_{+} \\ \alpha(H) s_{c} \in \pi / 2(\mathbb{Z}-2 \mathbb{Z})}} Y_{\alpha}+\sum_{\substack{\alpha \in R_{-} \\ \alpha(H) s_{c} \in \pi \mathbb{Z}-0}} Z_{\alpha}: Y_{\alpha} \in \mathbf{k}_{\alpha}^{-}, Z_{\alpha} \in \mathbf{k}_{\alpha}^{+}\right.\right.$and $\left.s_{c}>0\right\}$

describes all Jacobi fields along $\gamma=\gamma_{H}$ where $\gamma$ is a geodesic issuing from $p=\operatorname{Exp} H_{0}$ with initial tangent $H$ lying in a maximal abelian subalgebra $\mathbf{h}$ of $m^{-}$. By Proposition $2.3 \gamma\left(s_{f}\right)$ is a first conjugate point of $M^{+}(p)$ along $\gamma$ if and only if one of the following conditions is satisfied.

For all $\alpha$ such that $\alpha\left(H_{0}\right) \in \pi / 2(\mathbb{Z}-2 \mathbb{Z}), \alpha(H s)<\pi / 2$ if $s \in\left(0, s_{f}\right)$ and for all $\alpha$ such that $\alpha\left(H_{0}\right) \in \pi \mathbb{Z}, \alpha(H s)<\pi$ if $s \in\left(0, s_{f}\right)$, and either there exists an $\alpha$ with $\alpha\left(H_{0}\right) \in \pi / 2(\mathbb{Z}-2 \mathbb{Z})$ such that $\alpha\left(H s_{f}\right)=\pi / 2$, or there exists an $\alpha$ with $\alpha\left(H_{0}\right) \in \pi \mathbb{Z}$ such that $\alpha\left(H s_{f}\right)=\pi$. Restricting our attention to a positive Weyl chamber $C_{p}$ we have that $\alpha\left(H s_{f}\right) \leq \beta\left(H s_{f}\right)$ if $\alpha<\beta, \alpha$ and $\beta$ positive. We now consider the three possible descriptions for $M^{+}(p)$. 
Case I. $H_{0}=1 / 2 H_{i}$ with $n_{i}=1$ and $M^{+}(p)=K \operatorname{Exp} 1 / 2 H_{i}$.

In this case $\widetilde{\alpha}\left(1 / 2 H_{i}\right)=\sum n_{i} \alpha_{i}\left(1 / 2 H_{i}\right)=\pi / 2$ and therefore $\widetilde{\alpha} \in R_{+}$and $\widetilde{\alpha}(H s) \geq \alpha(H s)$ for all $\alpha \in R(M)$ and for all $s \in\left(0, s_{f}\right)$. If $\widetilde{\alpha}(H s)<\pi / 2$, then $\alpha(H s) \leq \widetilde{\alpha}(H s)<\pi / 2<\pi$ and therefore the first tangent conjugate locus of $M^{+}(p)$ in $C_{p}$ is given by $(\widetilde{\alpha})^{-1}(\pi / 2) \cap C_{p}$.

Case II. $H_{0}=1 / 2 H_{i}$ with $n_{i}=2$.

In this case $\widetilde{\alpha} \in R_{-}$since if $H_{0}=1 / 2 H_{i}$ with $n_{i}=2, \widetilde{\alpha}\left(1 / 2 H_{i}\right)=$ $\sum n_{i} \alpha_{i}\left(1 / 2 H_{i}\right)=\pi$. We now show that if $\widetilde{\beta}$ is the highest root in $R_{+}$, then $2 \widetilde{\beta} \geq \widetilde{\alpha}$ and therefore if $\widetilde{\alpha}\left(H s_{f}\right)=\pi$, we have that $\widetilde{\beta}\left(H s_{f}\right) \geq \pi / 2$ so that the first tangent conjugate locus of $M^{+}(p)$ in $C_{p}$ is given by $(\tilde{\beta})^{-1}(\pi / 2) \cap C_{p}$. In order to show that $2 \widetilde{\beta} \geq \widetilde{\alpha}$ we consider the cases where $M$ is of classical type and exceptional type separately. We assume first that $M$ is classical and $R(M) \neq D_{r}$, then $\left\langle\alpha_{i}, \alpha_{i+1}\right\rangle<0$ and therefore $\alpha_{i}+\alpha_{i+1} \in R(M)$, $\left\langle\alpha_{i-1}, \alpha_{i}+\alpha_{i+1}\right\rangle=\left\langle\alpha_{i-1}, \alpha_{i}\right\rangle+0<0$ and therefore $\alpha_{i-1}+\alpha_{i}+\alpha_{i+1} \in R(M)$. Continuing inductively we get that $\alpha_{1}+\cdots+\alpha_{r} \in R(M)$. If $R(M)=D_{r}$ we apply the above argument to get that $\alpha_{1}+\cdots+\alpha_{r-1} \in R(M)$, then $\left\langle\alpha_{1}+\cdots+\right.$ $\left.\alpha_{r-2}+\alpha_{r-1}, \alpha_{r}\right\rangle=\left\langle\alpha_{r-2}, \alpha_{r}\right\rangle<0$ and we again see that $\alpha_{1}+\cdots+\alpha_{r} \in R(M)$. Applying Corollary 2.3 we see that $\alpha_{1}+\cdots+\alpha_{r} \in R_{+}$, and since in the expression $\widetilde{\alpha}=\sum n_{i} \alpha_{i}$ all $n_{i} \leq 2$ when $M$ is of classical type, but not of type $B C$, we have that $2 \tilde{\beta} \geq \widetilde{\alpha}$ as linear forms on $C_{p}$. We next note that the roots in $R_{+}$ are the restrictions to $\mathbf{h}$ of the weights of the linear isotropy representation of $G^{-}$on the tangent space $m^{+}+k^{-}$to the symmetric space $G / G^{-}$, and therefore $\widetilde{\beta}$ is the dominant weight of this representation. Therefore in the case where $M$ is of exceptional type or $B C$, we merely read the $G^{-}$off the list of Nagano and Chen [N-C, 1] and since the dominant weights $\tilde{\beta}$ have been computed by Cartan $[\mathrm{C}, 1]$ we see that $2 \widetilde{\beta} \geq \widetilde{\alpha}$ and the theorem follows.

Note the same argument gives the following proposition.

Proposition 2.5. (1) $F L\left(F\left(s_{p}, M\right)(0), M\right)=K, \operatorname{Exp}\left\{(\widetilde{\alpha})^{-1}(\pi / 2) \cap C\right\}$ if $p=$ $\operatorname{Exp} 1 / 2 H_{i}$ with $n_{i}=1$.

(2) $F L\left(F\left(s_{p}, M\right)(0), M\right)=K, \operatorname{Exp}\left\{(\tilde{\beta})^{-1}(\pi / 2) \cap C\right\}$ if $p=\operatorname{Exp} 1 / 2 H_{i}$ with $n_{i}=2$, where $\widetilde{\alpha}$ is the highest root of $M, \widetilde{\beta}$ is the highest root in $R_{+}$ and $C$ is a closed positive Weyl chamber at 0 .

Corollary $2.6[\mathrm{~S}, 1],[\mathrm{T}, 1] . F L(\{0\}, M)=K, \operatorname{Exp}\left\{(\widetilde{\alpha})^{-1}(\pi) \cap C\right\}$ where $C$ is a closed positive Weyl chamber at 0 , and $\tilde{\alpha}$ is the highest root of $M$.

Proof. We apply Theorem 1.2 with $t=s_{0}$ and $M^{t}=\{0\}$ and therefore by Corollary $1.3 \mathscr{J}^{\gamma}=\left\{\left(\sum Y_{\alpha}\right)_{\mid \gamma}: Y_{\alpha} \in k_{\alpha}, \alpha \in R(M)\right.$ and $\alpha\left(H s_{c}\right) \in \pi \mathbb{Z}-$ $0\}$ describes all Jacobi fields along $\gamma=\gamma_{H}$ which vanish at 0 and for some parameter value $s_{c}>0$, the result now follows.

In this section we will discuss the first conjugate locus of an arbitrary $\mathrm{M}^{-}(p)$, and the applications of our theory to computing homology. In our discussion of $F L\left(M^{-}(p), M\right)$ we will denote by $M^{+}$the copy of $M^{+}(p)$ at 0 , that is $M^{+}=F\left(s_{p}, M\right)(0)$. Recall that $M^{-}(p)=M^{-}=G^{-}(0)=G^{-}(p)$ where $p$ 
is the antipodal point on the circle defining the pair $\left(M^{+}(p), M^{-}(p)\right)$. We denote by $\mathbf{g}^{+}$the Lie subalgebra $\mathbf{k}^{+}+\mathbf{m}^{+}$, and by $G^{+}$the corresponding connected Lie subgroup of $G$. Since $\mathbf{m}^{+} \cong \mathbf{k}^{-}$we have that $\mathbf{k} \cong \mathbf{k}^{+}+\mathbf{m}^{+}$, and $\mathbf{m}^{+}+\mathbf{m}^{-}=\mathbf{m} \cong \mathbf{k}^{-}+\mathbf{m}^{-}$. Since $\left[\mathbf{k}^{+}+\mathbf{m}^{+}, \mathbf{k}^{-}+\mathbf{m}^{-}\right] \subset \mathbf{k}^{-}+\mathbf{m}^{-}$we see that the adjoint representation of $\mathbf{k}^{+}+\mathbf{m}^{+}$on $\mathbf{k}^{-}+\mathbf{m}^{-}$is isomorphic to the linear isotropy representation of $K \cong G^{+}$on the tangent space to $M$ at 0 . Choosing a maximal abelian subalgebra $\mathbf{h}^{+}$in $\mathbf{m}^{+}$the decompositions of Lemma 1.1 at 0 with respect to $t=\operatorname{ad}\left(s_{p}\right) \operatorname{ad}\left(s_{0}\right)$ are:

$$
\mathbf{m}^{+}=\mathbf{h}^{+}+\sum_{\alpha \in R} \mathbf{m}_{\alpha}^{+}, \quad \mathbf{m}^{-}=\mathbf{m}_{0}^{-}+\sum_{\lambda \in \Lambda} \mathbf{m}_{\lambda}^{-}
$$

where $R=R\left(M^{+}\right)$are the roots of the symmetric space $M^{+}$and $\Lambda$ is the set of the weights of the adjoint representation of $\mathbf{g}^{+}$on $\mathbf{m}^{-}+\mathbf{k}^{-}$restricted to $\mathbf{h}^{+}$. By the above discussion these weights are the same as those of the linear isotropy representation of $K$ on $T_{0} M$ since the representations are isomorphic, we note that the dominant weights of these representations have been calculated by Cartan [C, 1]. Again we restrict our attention to the case where this representation is absolutely irreducible.

Lemma 3.1. Let $\tilde{\lambda}$ be the dominant weight in $\Lambda$, and let $\tilde{\alpha}$ be the highest root of $M^{+}$, then $2 \tilde{\lambda} \geq \tilde{\alpha}$.

Proof. Consider the following composition of maps, where the first map is the bracket product and the second is just the obvious projection.

$$
\mathbf{m}^{-}+\mathbf{k}^{-} \otimes \mathbf{m}^{-}+\mathbf{k}^{-} \rightarrow \mathbf{k}^{+}+\mathbf{m}^{+} \rightarrow \mathbf{m}^{+} .
$$

Since $\mathrm{m}^{+}$is a direct sum of nonzero root spaces with respect to a maximal abelian subalgebra $\mathbf{h}^{-}$of $\mathbf{m}^{-}$the first map has in its image vectors with arbitrary $\mathbf{m}^{+}$component, and therefore the composite is surjective. Taking the root space decomposition of $\mathbf{m}^{+}$with respect to $\mathbf{h}^{+}$, and the weight space decomposition of $\mathbf{m}^{-}+\mathbf{k}^{-}$described above, representation theory then tells us that every root is a sum of two weights. However each weight $\lambda \in \Lambda$ is of the form $\lambda=\tilde{\lambda}-\sum m_{i} \alpha_{i}$ where the $\alpha_{i}$ 's are positive roots of $M^{+}$(see [H, 2, Chapter 5]). We now have $\tilde{\alpha}=\tilde{\lambda}-\sum m_{j} \alpha_{j}+\tilde{\lambda}-\sum n_{i} \alpha_{i} \leq 2 \tilde{\lambda}$.

Theorem 3.2. The first conjugate locus of $M^{-}(p)$ in $M$ has the following description, $F L\left(M^{-}(p), M\right)=G^{-} \operatorname{Exp}\left\{(\tilde{\lambda})^{-1}(\pi / 2) \cap C\right\}$ where $C$ is a positive Weyl chamber in $h^{+}$at 0 .

Proof. Let $\gamma_{H}=\gamma$ be a geodesic normal to $M^{-}(p)$ at 0 with initial tangent $H$ which we may assume to lie in a closed positive Weyl chamber $C$ in $\mathbf{h}^{+}$. Corollary 1.3 then tells us that

$$
\mathscr{J}^{\gamma}=\left\{\left(\sum_{\substack{\lambda \in \Lambda \\ \lambda\left(H s_{c}\right) \in \pi / 2(\mathbb{Z}-2 \mathbb{Z})}} X_{\lambda}+\sum_{\substack{\alpha \in R \\ \alpha\left(H s_{c} \in \in(\mathbb{Z}-0)\right.}} Y_{\alpha}: X_{\lambda} \in m_{\lambda}^{-}, Y_{\alpha} \in k_{\alpha}^{+}, s_{c}>0\right\}\right.
$$

describes all Jacobi fields along $\gamma$ satisfying the necessary initial conditions for $M^{-}(p)$ and vanishing for some parameter value $s_{c}>0$. Thus $\gamma\left(s_{f}\right)$ being a first conjugate point of $M^{-}(p)$ along $\gamma$ is equivalent to one of the following conditions being satisfied. 
For all $\lambda \in \Lambda$ we have $\lambda(H s)<\pi / 2$ for $s \in\left(0, s_{f}\right)$, and for all $\alpha \in$ $R \alpha(H s)<\pi$ for $s \in\left(0, s_{f}\right)$, and either there exists a $\lambda \in \Lambda$ with $\lambda\left(H s_{f}\right)=$ $\pi / 2$ or there exists an $\alpha \in R$ with $\alpha\left(H s_{f}\right)=\pi$. Since $H \in C$ we may use the previous lemma to conclude that the first conjugate point comes from the Jacobi fields $X_{\tilde{\lambda}}$ and therefore the first tangent conjugate locus of $M^{-}(p)$ in $M$ intersected with the positive Weyl chamber is given by $(\tilde{\lambda})^{-1}(\pi / 2) \cap C$.

Corollary 3.3. If $M^{+}(p)$ has rank one then $F L\left(M^{-}(p), M\right)$ is a submanifold. Proof. Consider the hyperplane $(\tilde{\lambda})^{-1}(\pi / 2)=\left\{H \in \mathbf{h}^{+} \mid \tilde{\lambda}(H)=\pi / 2\right\}$, since $\mathbf{h}^{+}$is one dimensional $=\mathbb{R} H_{0}$ where $H_{0}$ may be taken such that $\tilde{\lambda}\left(H_{0}\right)=1$. Therefore $H$ lies on the above hyperplane if and only if $H=\pi / 2 H_{0}$, and Theorem 3.2 tells us that $F L\left(M^{-}(p), M\right)=G^{-}\left(x_{0}\right)$ where $x_{0}=\operatorname{Exp} \pi / 2 H_{0}$ and is a submanifold.

Definition. Let $N$ be a closed connected submanifold of a complete connected Riemannian manifold $M$. Then $x \in M$ is said to be a cut point of $N$ along $\gamma$ if $\gamma(0)=x, \gamma\left(t_{1}\right) \in N, t_{1}>0$ and the following two conditions hold.

(1) For all $t \in\left(0, t_{1}\right], \gamma$ restricted to $\left[t, t_{1}\right]$ is a shortest geodesic from $\gamma(t)$ to $N$, and

(2) for all $t<0, \gamma$ restricted to $\left[t, t_{1}\right]$ is not a shortest geodesic from $\gamma(t)$ to $N$. The cut locus of $N$ in $M$, denoted by $\operatorname{CL}(N, M)$ is then defined as follows $\mathrm{CL}(N, M)=\{x \in M \mid x$ is a cut point of $N$ along some geodesic $\}$.

Proposition 3.4. If the pair $\left(M^{+}(p), M^{-}(p)\right)$ is such that $M^{+}(p)$ has rank one, then the first conjugate locus of $M^{-}(p)$ and the cut locus of $M^{-}(p)$ agree, that is, $F L\left(M^{-}(p), M\right)=\mathrm{CL}\left(M^{-}(p), M\right)$.

Proof. Let $x_{0}$ be as in the previous corollary, that is $G^{-}\left(x_{0}\right)=F L\left(M^{-}(p), M\right)$, and $x_{0} \in A^{+}$a maximal torus in $M^{+}$at 0 . It can be checked that $x_{0} \neq 0$. Since $M^{+}$has rank one $A^{+}$is just a circle which we will denote by $\gamma$. Suppose $\gamma_{1}$ is a shortest geodesic from $x_{0}$ to $M^{-}(p)$, and therefore $\gamma_{1}$ is normal to $M^{-}(p)=G^{-}(0)$ at $y$ say. Applying an element $b \in G^{-}$such that $b(y)=0$ to $\gamma_{1}$ gives us a geodesic at 0 which is again normal to $M^{-}(p)$ and is therefore contained in $M^{+}$. We can therefore apply an element $l \in K_{(1)}^{+} \subset G^{-}$to $b \gamma_{1}$ so that $l b \gamma_{1} \subset \gamma$. Since $l b\left(x_{0}\right)$ is in the same $G^{-}$orbit as $x_{0}$ Proposition 1.10 tells us it is a conjugate point of $M^{-}(p)$. However since $x_{0}$ is the first conjugate point of $M^{-}(p)$ along $\gamma$ we have that $l b\left(x_{0}\right)=x_{0}$ and hence the first conjugate locus of $M^{-}(p)$ is contained in the cut locus of $M^{-}(p)$ because $\gamma_{1}$ was a shortest geodesic to $M^{-}(p)$. Suppose now that $q$ is a cut point of $M^{-}(p)$ along some geodesic, then we again bring it into the maximal torus $\gamma$. Now from the above argument the point that $q$ goes to in $\gamma c$ does not come before $x_{0}$, and therefore it must actually be $x_{0}$ so the corollary is proved.

We now illustrate the preceding theory using the example of $C I(2)=G_{2}^{0}\left(\mathbb{R}^{5}\right)$ the Grassmann manifold of oriented 2 planes in $\mathbb{R}^{5}$. We will use the theory of Morse as applied by Bott [B, 1] to compute the homology of this space. The same analysis can be applied to other spaces with a rank one $M^{+}(p)$. These include most classical spaces and exceptional spaces such as $F_{4}, F I$, and $E I V$. Also some exceptional spaces have other fixed point sets of involutions which have normal spaces of rank one to which we can apply the same theory. 
Example 1. $M=G_{2}^{0}\left(\mathbb{R}^{5}\right)$. The pairs $\left(M^{+}(p), M^{-}(p)\right)$ have been calculated for all symmetric spaces by Nagano and Chen [N-C, 1], and we will use their descriptions. We will use $M^{-}(p)=G_{2}^{0}\left(e_{1} \wedge e_{2} \wedge e_{3} \wedge e_{4}\right) \cong S^{2} \times S^{2}$ where the $e_{i}$ 's are the standard basis for $\mathbb{R}^{5} G=S O(5), K=S O(2) \times S O(3)$. We take $0=e_{1} \wedge e_{2}$ as our origin and $p=e_{3} \wedge e_{4}$. A direct computation then gives the descriptions $M^{+}(p)=G_{2}^{0}\left(e_{3} \wedge e_{4} \wedge e_{5}\right) \cong S^{2}, G^{-}=S O(4) \times S O(1)$. We will use $\gamma(t)=e_{3} \wedge\left(\cos t e_{4}+\sin t e_{5}\right)$ as our torus at $p=\gamma(0)$. For $b \in G^{-} b \gamma(t)=b e_{3} \wedge\left(\cos t b e_{4}+\sin t b e_{5}\right)$ and therefore $b \gamma(t)=\gamma(t)$ if and only if $b e_{3} \wedge \cos t b e_{4}-e_{3} \wedge \cos t e_{4}=-b e_{3} \wedge \sin t e_{5}+e_{3} \wedge \sin t e_{5}$. Since the right-hand side of this equation contains an $e_{5}$ term but the left-hand side does not we see that these two 2 planes are orthogonal and therefore equality holds if and only if both are zero. This is equivalent to the following conditions being satisfied: $\cos t b\left(e_{3} \wedge e_{4}\right)=\cos t e_{3} \wedge e_{4}$, and $\sin t\left(b e_{3} \wedge e_{5}\right)=\sin t\left(e_{3} \wedge e_{5}\right)$. We may assume that $\sin t \neq 0$ since in that case $\gamma(t) \in M^{-}(p)$. Now $b \gamma(t)=$ $\gamma(t)$ implies that $b e_{3}=e_{3}$ and $\cos t e_{3} \wedge b e_{4}=\cos t\left(e_{3} \wedge e_{4}\right)$ and therefore we get a larger isotropy subgroup giving us a singular $G^{-}$orbit if and only if $\cos t=0$ (excluding $M^{-}(p)$ of course). By Theorem 1.2 and the fact that there is only one singular $G^{-}$orbit apart from $M^{-}(p)$ itself, we have that this singular orbit is the first conjugate locus of $M^{-}(p)$ and therefore by Proposition 3.4 it is also the cut locus of $M^{-}(p)$. Now if $\cos t=0$ then $b \in S O\left(e_{1} \wedge e_{2} \wedge e_{4}\right)$ $\cong S O(3)$ and $G^{-}(\gamma(t))=S O(4) / S O(3) \cong S^{3}$. We therefore have that $\mathrm{CL}\left(M^{-}(p), M\right) \cong S^{3}$. Note that it is a manifold. We can now use (essentially) the square of the distance from $M^{-}(p)$ as a Morse function on $M$ whose critical submanifolds are just $M^{-}(p)$ and the cut locus $S^{3}$. Since the null space of the hessian at critical points on $S^{3}$ and $M^{-}(p)$ are just the tangent spaces at these points to the appropriate submanifold, we have that the critical submanifolds are nondegenerate. We now have from the work of Bott [B, 1] that $M=M^{-}(p) \cup \xi_{3}\left(S^{3}\right)$ where $\xi_{3}\left(S^{3}\right)$ is a plane bundle over $S^{3}$. We now compute how to attach this cell to $M^{-}(p)$. To do so we consider the flow lines of the gradient of our Morse function that is the geodesics normal to $M^{-}(p)$. Take a cellular decomposition of $S^{3}=e^{0} \cup e^{3}$ where $e^{0}=e_{1} \wedge e_{5}$, the appropriate torus passing through $e^{0}$ is given by $e_{1} \wedge\left(\cos t e_{2}+\sin t e_{5}\right)$, and it intersects $M^{-}(p)$ when $\sin t=0$. Applying the isotropy subgroup of $e_{1} \wedge e_{5}$ to $e_{1} \wedge\left(\cos 0 e_{3}+\sin 0 e_{5}\right)$ we see that the intersection of the flow lines into $e_{1} \wedge e_{5}$ with $M^{-}(p)$, that is $\operatorname{bd}\left(e^{3}\right)$, is given by $\left\{e_{1} \wedge b e_{2} \mid b \in S O(1) \times S O(3) \times S O(1)\right\} \cong G_{1}^{0}\left(e_{2} \wedge e_{3} \wedge e_{4}\right) \cong S^{2}$. Note if $b e_{2}=-e_{2}$ we are at the point $e_{1} \wedge-e_{2}$ which is the other $M^{+}$of $M$, called the pole of $C I(2)$. This point is antipodal to 0 on the product of Helgason spheres which give $M^{-}(p)\left(M^{-}(p)=S^{2} \times S^{2}\right)$, and therefore $\operatorname{bd}\left(e^{3}\right) \cong S^{2}$ sits diagonally in the product of Helgason spheres. This means that $e^{3}$ bounds one of these spheres but not both as they are independent. The cell $e^{3} \times e^{3}$ is trivially a cycle since its boundary has dimension five and $M^{-}(p)$ has dimension four. These observations now give the following description of the homology of $C I(2)$.

$$
H_{*}(C I(2), \mathbb{Z}) \cong \mathbb{Z}[0]+\mathbb{Z}\left[S^{2}\right]+\mathbb{Z}\left[M^{-}(p)\right]+\mathbb{Z}[M]
$$

that is

$$
H_{*}(C I(2), \mathbb{Z}) \cong H_{0}(C I(2), \mathbb{Z})+H_{2}(C I(2), \mathbb{Z})+H_{4}(C I(2), \mathbb{Z})+H_{6}(C I(2), \mathbb{Z})
$$




\section{Example 2.}

$$
\mathrm{CL}\left(\operatorname{Spin}(9), F_{4}\right)=(\operatorname{Spin}(9) \times \operatorname{Spin}(9)) / \operatorname{Spin}(8) .
$$

Since $\operatorname{Spin}(9)$ is an $M^{-}$of $F_{4}$ with corresponding $M^{+}=F I I$ which is a space of rank one we have by Proposition 3.4 that the cut locus is the same as the first conjugate locus and is therefore a $G^{-}$orbit of a point $x_{0}$ in the maximal torus of the copy of $M^{+}$at the identity element of the group $F_{4}$. The $G^{-}$in this case is $\operatorname{Spin}(9) \times \operatorname{Spin}(9)$ and its elements $(b, c)$ act on a point $x \in F_{4}$ via $x \rightarrow b x c^{-1}$. Let $x_{0}=\gamma\left(t_{0}\right)$ be a point in the maximal torus of the above $F I I$ and let $\gamma(t)$ be a minimizing geodesic from $x_{0}$ to $\operatorname{Spin}(9)$ which intersects $\operatorname{Spin}(9)$ at the identity element at time $t=0$. We can always find such an $x_{0}$ since $\operatorname{Spin}(9)$ is the $G^{-}$orbit of the identity element and any minimizing geodesic to $\operatorname{Spin}(9)$ will be normal to $\operatorname{Spin}(9)$, hence will still be normal when brought to the identity by an element of $G^{-}$. Since the $F I I$ is totally geodesic this geodesic will lie in the $F I I$ and will therefore intersect its maximal torus at some point $x_{0}$. We now note that if there exists an element $(b, c) \in G^{-}$such that $b x_{0} c^{-1}=x_{0}$ with $b \neq c$ then $x_{0} \in \mathrm{CL}\left(\operatorname{Spin}(9), F_{4}\right)$, as the element $(b, c)$ will move $\gamma(0)$ which is the identity, but will fix $x_{0}$, giving us at least two minimizing geodesics to $\operatorname{Spin}(9)$ from $x_{0}$. We have therefore that the cut locus is the $G^{-}$orbit of such an $x_{0}$. We will now determine such $x_{0}$ 's. Let $\sigma_{I I}$ be the involution defining the space $F I I$. Then $\operatorname{Spin}(9)=F\left(\sigma_{I I}, F_{4}\right)$ and $F I I$ containing the identity element is given by $F I I=F\left(\sigma_{I I} \circ s_{1}, F_{4}\right)$. We see therefore that $X_{0} \in F I I$ if and only if $\sigma_{I I}\left(x_{0}\right)=x_{0}^{-1}$, so that $b x_{0} c^{-1}=x_{0}$ if and only if $b x_{0} c^{-1}=x_{0}^{-1}$. If $y=b x_{0} b^{-1}$ then we see that if $x_{0} \in F I I$ is such that $b x_{0} c^{-1}=x_{0}$ then $x_{0}^{2}=b x_{0} c^{-1} x_{0}=b x_{0} b^{-1} b c^{-1} x_{0}=y b c^{-1} x_{0}=y b x_{0}^{-1} b^{-1} x_{0}=y^{2}$. We have therefore that $x_{0}^{2}=\gamma\left(2 t_{0}\right)=b \gamma\left(2 t_{0}\right) b^{-1}=y^{2}$. Since $x \neq y$ this can only happen if $x^{2}$ sits on the $M^{+}$of the space $F I I$ which by the list of Nagano and Chen [N-C, 1] is an $S^{8}$ with corresponding $M^{-}$which is also an $S^{8}$. We have therefore that $x_{0}$ and $y$ sit on the equator $S^{7}$ of the latter $S^{8}$. Conversely if $y$ and $x_{0}$ sit on this $S^{7}$ there exists $b \in \operatorname{Spin}(8)$ such that $y=b x_{0} b^{-1}$. Since $\sigma_{I I}\left(y^{-1} x_{0}\right)=y x_{0}^{-1}$ and since $y^{2}=x_{0}^{2}$ we have that $\sigma_{I I}\left(y^{-1} x_{0}\right)=y^{-1} x_{0}$ and therefore $y^{-1} x_{0}$ is an element of $\operatorname{Spin}(9)$. Thus we have the existence of a unique element $c \in \operatorname{Spin}(9)$ such that $y^{-1} x_{0}=b c$, that is $x_{0}=y b c=b x_{0} b^{-1} b c=b x_{0} c$ with $b \neq c$. We now have that the isotropy subgroup of such $x_{0}$ 's is $\operatorname{Spin}(8)$ thus giving the desired result.

As in the previous example we get a Morse function with only two critical submanifolds corresponding to the max and min. The actual attaching maps are more difficult to compute but the following inequalities for any coefficient field $K$ are immediate from the Morse-Bott inequalities.

\section{Proposition 3.5.}

$$
b_{i}\left(F_{4} ; K\right) \leq b_{i}(\operatorname{Spin}(9) ; K)+b_{i-8}(\operatorname{Spin}(9) \times \operatorname{Spin}(9) / \operatorname{Spin}(8), K) .
$$

Example 3.

$$
\mathrm{CL}\left(G_{4}^{0}\left(\mathbb{R}^{9}\right), F I\right)=\mathrm{CL}\left(\operatorname{Spin}(9), F_{4}\right) \cap F I .
$$

Let $Q: F I \rightarrow F_{4}$ be the quadratic representation. Since $F I$ has no poles $Q$ is an embedding $[\mathrm{N}-\mathrm{C}, 1]$ and we see from the list of Nagano and Chen $[\mathrm{N}-\mathrm{C}]$, 
by noting that $M^{-}$corresponding to $\operatorname{Spin}(9)$ in $F I$, that $Q^{-1}(\operatorname{Spin}(9))=$ $G_{4}^{0}\left(\mathbb{R}^{9}\right)$. Again we obtain the usual Morse inequalities.

\section{REFERENCES}

[A, 1] W. Ambrose, The index theorem in Riemannian geometry, Ann. of Math. (2) 73 (1961), 49-86.

[B, 1] R. Bott, Non degenerate critical manifolds, Ann. of Math. (2) 60 (1958), 242-261.

[B-S, 1] R. Bott and H. Samelson, An application of the theory of Morse to symmetric spaces, Amer. J. Math. 78 (1958), 964-1028.

[Br, 1] G. Bredon, Compact transformation groups, Academic Press, New York, 1972.

[C, 1] E. Cartan, Sur certain formes Riemanniennes remarquable des geometrie a groups fondamental simple, Ann. Sci. École Norm. Sup. 44 (1929), 345-467.

[Cr, 1] R. Crittenden, Minimum and conjugate points in symmetric spaces, Canad. J. Math. 14 (1962), 320-328.

$[\mathrm{H}, 1] \mathrm{S}$. Helgason, Differential geometry, Lie groups, and symmetric spaces, Academic Press, New York, 1978.

[H, 2] - Groups and geometric analysis, Academic Press, New York, 1984.

$[\mathrm{K}, 1]$ S. Kobayashi, Fixed points of isometries, Nagoya Math J. 13 (1958), 63-68.

[N-C, 1] T. Nagano and B. Y. Chen, Totally geodesic submanifolds of symmetric spaces. II, Duke Math. J. 45 (1978), 405-425.

[S, 1] T. Sakai, On the structure of cut loci in compact Riemannian symmetric spaces, Math. Ann. 235 (1978), 129-148.

[T, 1] M. Takeuchi, On conjugate loci and cut loci of compact symmetric spaces. I, Tsukuba J. Math. 2 (1977), 35-68.

[W, 1] Y. C. Wong, Conjugate loci in Grassmann manifolds, Bull. Amer. Math. Soc. 74 (1968), 204-245.

Dublin Institute of Technology, Kevin Street, Dublin 8, Ireland

Current address: Department of Mathematics, University College, Galway, Ireland

E-mail address: matburns@bodkin.ucg.ie 